\title{
Higher dietary protein, hsCRP levels and polygenic risk scores of genetically predisposed overweight and obese Malaysian adults
}

\author{
P.Y. Tan ${ }^{1}$, F. Amini ${ }^{2}$ and S.R. Mitra ${ }^{3}$ \\ ${ }^{1}$ School of Food Science and Nutrition, Faculty of Environment, University of Leeds, Leeds, UK, \\ ${ }^{2}$ School of Healthy Aging, Medical Aesthetics \& Regenerative Medicine, UCSI University, KL Campus, Malaysia and \\ ${ }^{3}$ School of Biosciences, Faculty of Science and Engineering, University of Nottingham Malaysia, Semenyih, Selangor \\ Darul Ehsan, Malaysia
}

Evidence suggests that dietary nutrients may interact with gene variants and modulate inflammatory status in obese individuals ${ }^{(1)}$. This study, aimed to investigate the combined effect of FTO (rs9930506, rs9930506 and rs9932754) and ADRB2 (rs1042713) gene variants on high-sensitive $\mathrm{C}$ reactive protein (hsCRP) levels using polygenic risk scores (PRS). Modulatory effects of dietary nutrients on the above associations were investigated as well.

128 overweight and obese Malaysian (57 Malay, 24 Chinese and 47 Indian) adults were recruited. Dietary, physical activity, anthropometric and blood biochemical parameters were collected. Elevated hsCRP level was defined as hsRCP $>3 \mathrm{mg} / \mathrm{L}$, indicating higher inflammatory status and risk of cardiovascular events ${ }^{(2)}$. The risk allele of each SNP (single nucleotide polymorphism) was captured from published GWAS studies ${ }^{(3-4)}$, including FTO rs9930501 (G allele), rs9930506 (G allele) and rs9932754 (C allele) and ADRB2 rs1042713 (G allele). The odds ratio (OR) of obesity for each SNP was computed. Genetic predisposition to obesity was assessed by PRS. PRS was computed as the sum of individual's risk alleles across the 4 SNPs, weighted by the effect size of each risk allele using their respective natural $\log$ of OR for obesity. Participants were stratified into tertiles of first (0-0.64), second (0.65-3.59) and third (3.60-8.18) PRS. Ethical approval was obtained from the Science and Engineering Research Ethics Committee, University of Nottingham Malaysia. Written informed consent was obtained from all participants. Statistics: Binary logistic regression was performed to determine the effect of PRS on the odds of elevated hsCRP level. Gene-diet interaction on hsCRP level was conducted by using general linear model after adjusting for covariates. Dietary protein was dichotomized to 2 groups using the median value of $14 \% /$ day, for analysis.

$51 \%$ of our study participants were found to have elevated hsCRP levels. hsCRP levels were significantly associated with higher body weight $(p=0.025)$, BMI $(p=0.003)$, WC $(p<0.001)$, WHR $(p=0.033)$, fat mass $(p=0.001)$ and percent body fat $(p<0.001)$. Logistic regression revealed that second tertile (0.65-3.58) and third tertile (3.59-8.18) PRS were significantly associated with increased odds of elevated hsCRP level, $7.56(\mathrm{CI}=1.98-28.80$; adjusted $\mathrm{p}=0.003)$ and $3.87(\mathrm{CI}=1.10-13.60$; adjusted $\mathrm{p}=0.035)$ respectively, compared to the first tertile PRS (0-0.64). Higher number of Malaysian Indians were categorised under third tertile PRS ( $42 \%$ vs $32 \%$ and $21 \%),(\mathrm{p}=0.016)$. Gene-diet interaction analysis revealed that individuals in the third tertile PRS had significantly lower hsCRP levels $(4.61 \pm 1.3 \mathrm{mg} / \mathrm{L}$ vs $9.60 \pm 2.6 \mathrm{mg} / \mathrm{L}, \mathrm{p}=0.019)$ with consumption of high percent energy from dietary protein ( $\geq 14 \% /$ day), compared to low percent energy from dietary protein.

Highest PRS was significantly associated with increased odds of hSCRP. In individuals with high PRS, higher intake of dietary protein may alleviate inflammatory status of individuals and reduce hsCRP levels systemically.

\section{References}

1. Phillips CM (2013) Nutrients 5, 2-57.

2. Yousuf O, Mohanty BD, Martin SS, et al. (2013) J Am Coll Cardiol 62, 397-408.

3. Mitra SM, Tan PY \& Amini F (2018) J Hum Nutr Diet 31, 758-772.

4. Mitra SM, Tan PY \& Amini F (2019) J Nutr Metab e8718795. 